\title{
Patients with coronary heart disease, dilated cardiomyopathy and idiopathic ventricular tachycardia share overlapping patterns of pathogenic variation in cardiac risk genes
}

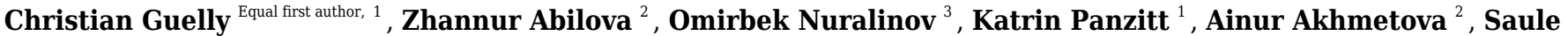 \\ Rakhimova $^{2}$, Ulan Kozhamkulov ${ }^{2}$, Ulykbek Kairov ${ }^{4}$, Askhat Molkenov ${ }^{4}$, Ainur Seisenova ${ }^{4}$, Slave Trajanoski ${ }^{1}$, \\ Gulzhaina Abildinova (Rashbayeva) ${ }^{3}$, Galina Kaussova ${ }^{5}$, Christian Windpassinger ${ }^{6}$, Joseph H Lee ${ }^{7}$, Zhaxybay \\ Zhumadilov $^{2}$, Makhabbat Bekbossynova ${ }^{3}$, Ainur Akilzhanova ${ }^{\text {Corresp. Equal first author, } 2}$ \\ ${ }^{1}$ Center of Medical Research, Medical University of Graz, Graz, Austria \\ 2 Laboratory of Genomic and Personalized Medicine, Center for Life Science, National Laboratory Astana, Nazarbayev University, Nur-Sultan, Kazakhstan \\ 3 National Research Cardiac Surgery Center, Nur-Sultan, Kazakhstan \\ 4 Laboratory of Bioinformatics and Systems Biology, Center for Life Sciences, National Laboratory Astana, Nazarbayev University, Nur-Sultan, Kazakhstan \\ 5 Kazakhstan medical university "KSPH", Almaty, Kazakhstan \\ 6 Institute of Human Genetics, Medical University of Graz, Graz, Austria \\ 7 Sergievsky Center - Taub Institute, Columbia University Medical Center, New York, United States \\ Corresponding Author: Ainur Akilzhanova \\ Email address: akilzhan ainur@mail.ru
}

Background. Ventricular tachycardia (VT) is a major cause of sudden cardiac death (SCD). Clinical investigations can sometimes fail to identify the underlying cause of VT and the event is classified as idiopathic (iVT). VT contributes significantly to the morbidity and mortality in patients with coronary artery disease (CAD) and dilated cardiomyopathy (DCM). Since mutations in arrhythmia-associated genes frequently determine arrhythmia susceptibility screening for disease-predisposing variants could improve VT diagnostics and prevent SCD in patients. Methods. 92 patients diagnosed with coronary heart disease (CHD), DCM, or iVT were included in our study. We evaluated genetic profiles and variants in known cardiac risk genes by targeted next generation sequencing (NGS) using a newly designed custom panel of 96 genes. We hypothesized that shared morphological and phenotypical features among these subgroups may have an overlapping molecular base. To our knowledge, this was the first study of the deep sequencing of 96 targeted cardiac genes in Kazakhstan. The clinical significance of the sequence variants was interpreted according to the guidelines developed by the American College of Medical Genetics and Genomics (ACMG) and the Association for Molecular Pathology (AMP) in 2015. The ClinVar and Varsome databases were used to determine the variant classifications. Results. Targeted sequencing and stepwise filtering of the annotated variants identified a total of 307 unique variants in 74 genes, totally 456 variants in the overall study group. We found 168 mutations listed in the Human Genome Mutation Database (HGMD) and another 256 
rare/unique variants with elevated pathogenic potential. There was a predominance of high- to intermediate pathogenicity variants in $L A M A 2, M Y B P C 3, M Y H 6, K C N Q 1, G A A$, and $D S G 2$ in CHD VT patients. Similar frequencies were observed in DCM VT, and iVT patients, pointing to a common molecular disease association. TTN, GAA, LAMA2, and MYBPC3 contained the most variants in the three subgroups which confirm the impact of these genes in the complex pathogenesis of cardiomyopathies and VT. The classification of 307 variants according to ACMG guidelines showed that 9 (2.9\%) variants could be classified as pathogenic, 9 (2.9\%) were likely pathogenic, 98 (31.9\%) were of uncertain significance, 73 (23.8\%) were likely benign, and 118 (38.4\%) were benign. CHD VT patients carry rare genetic variants with increased pathogenic potential at a comparable frequency to DCM VT and IVT patients in genes related to sarcomere function, nuclear function, ion flux, and metabolism. Conclusions. In this study we showed that in patients with VT secondary to coronary artery disease, DCM, or idiopathic etiology multiple rare mutations and clinically significant sequence variants in classic cardiac risk genes associated with cardiac channelopathies and cardiomyopathies were found in a similar pattern and at a comparable frequency. 
1 Patients with coronary heart disease, dilated

2 cardiomyopathy and idiopathic ventricular

3 tachycardia share overlapping patterns of pathogenic

4 variation in cardiac risk genes

5

6

7

12

Christian Guelly ${ }^{1 \S}$, Zhannur M. Abilova ${ }^{2}$, Omirbek M. Nuralinov ${ }^{3}$, Katrin Panzitt ${ }^{1}$, Ainur Zh. Akhmetova $^{2}$, Saule E. Rakhimova ${ }^{2}$, Ulan A. Kozhamkulov², Ulykbek E. Kairov ${ }^{4}$, Askhat B. Molkenov $^{4}$, Ainur, T. Seisenova ${ }^{4}$, Slave Trajanoski ${ }^{1}$, Gulzhaina S. Rashbayeva ${ }^{3}$, Galina K. Kaussova $^{5}$, Christian Windpassinger ${ }^{6}$, Joseph H. Lee ${ }^{7}$, Zhaxybay Sh. Zhumadilov ${ }^{2}$, Makhabbat S. Bekbossynova ${ }^{3}$ and Ainur R. Akilzhanova ${ }^{28^{*}}$.

${ }^{1}$ Center of Medical Research, Medical University of Graz, Graz, Austria

${ }^{2}$ Laboratory of Genomic and Personalized Medicine, Center for Life Sciences, National Laboratory Astana, Nazarbayev University, Nur-Sultan, Kazakhstan

${ }^{3}$ National Research Cardiac Surgery Center, Nur-Sultan, Kazakhstan

${ }^{4}$ Laboratory of Bioinformatics and Systems Biology, Center for Life Sciences, National Laboratory Astana, Nazarbayev University, Nur-Sultan, Kazakhstan

${ }^{5}$ Kazakhstan Medical University "KSPH", Almaty, Kazakhstan

${ }^{6}$ Institute of Human Genetics, Medical University of Graz, Graz, Austria

${ }^{7}$ Sergievsky Center - Taub Institute, Columbia University Medical Center, New York, USA

$\S$ Equal contributors

*Corresponding Author:

Ainur Akilzhanova

$\mathrm{MD}, \mathrm{PhD}$, DSci, Associated Professor

Leading research scientist,

Head of Laboratory of Genomic and Personalized Medicine, Center for Life Sciences, PI "National Laboratory Astana"

JSC "Nazarbayev University", 53 Kabanbay Batyr ave, 010000, Nur-Sultan, Kazakhstan

Email address: akilzhanova@nu.edu.kz, akilzhan ainur@mail.ru

\section{Abstract}

Background. Ventricular tachycardia (VT) is a major cause of sudden cardiac death (SCD).

Clinical investigations can sometimes fail to identify the underlying cause of VT and the event is 
classified as idiopathic (iVT). VT contributes significantly to the morbidity and mortality in patients with coronary artery disease (CAD) and dilated cardiomyopathy (DCM). Since mutations in arrhythmia-associated genes frequently determine arrhythmia susceptibility screening for disease-predisposing variants could improve VT diagnostics and prevent SCD in patients.

Methods. 92 patients diagnosed with coronary heart disease (CHD), DCM, or iVT were included in our study. We evaluated genetic profiles and variants in known cardiac risk genes by targeted next generation sequencing (NGS) using a newly designed custom panel of 96 genes. We hypothesized that shared morphological and phenotypical features among these subgroups may have an overlapping molecular base. To our knowledge, this was the first study of the deep sequencing of 96 targeted cardiac genes in Kazakhstan. The clinical significance of the sequence variants was interpreted according to the guidelines developed by the American College of Medical Genetics and Genomics (ACMG) and the Association for Molecular Pathology (AMP) in 2015. The ClinVar and Varsome databases were used to determine the variant classifications.

Results. Targeted sequencing and stepwise filtering of the annotated variants identified a total of 307 unique variants in 74 genes, totally 456 variants in the overall study group. We found 168 mutations listed in the Human Genome Mutation Database (HGMD) and another 256 rare/unique variants with elevated pathogenic potential. There was a predominance of high- to intermediate pathogenicity variants in LAMA2, MYBPC3, MYH6, KCNQ1, GAA, and DSG2 in CHD VT patients. Similar frequencies were observed in DCM VT, and iVT patients, pointing to a common molecular disease association. TTN, GAA, LAMA2, and MYBPC3 contained the most variants in the three subgroups which confirm the impact of these genes in the complex pathogenesis of cardiomyopathies and VT. The classification of 307 variants according to ACMG guidelines showed that 9 (2.9\%) variants could be classified as pathogenic, 9 (2.9\%) were likely pathogenic, 98 (31.9\%) were of uncertain significance, 73 (23.8\%) were likely benign, and 118 (38.4\%) were benign. CHD VT patients carry rare genetic variants with increased pathogenic potential at a comparable frequency to DCM VT and iVT patients in genes related to sarcomere function, nuclear function, ion flux, and metabolism.

Conclusions. In this study we showed that in patients with VT secondary to coronary artery disease, DCM, or idiopathic etiology multiple rare mutations and clinically significant sequence variants in classic cardiac risk genes associated with cardiac channelopathies and cardiomyopathies were found in a similar pattern and at a comparable frequency.

\section{Introduction}


72

7317.5 million people died from cardiovascular diseases in 2012 and of these, 7.4 million deaths

74

75

76

77

78

79

80

81

82

83

84

85

86

87

88

89

90

91

92

93

94

95

96

97

98

99

100

101

102

103

104

105

106

107

108

109

110

111

112 were due to coronary heart disease (CHD), making it the number one cause of cardiac deaths, according to the WHO [1]. More than $75 \%$ of the cardiovascular disease (CVD) deaths occurred in low- and middle-income countries, with high rates in Kazakhstan, despite numerous state-run health programs. The age-standardized mortality rate of cardiovascular disorders in Kazakhstan is amongst the highest in the world. According to the latest WHO data published in 2018, there were 47,651 coronary heart disease deaths in Kazakhstan, amounting to $34.56 \%$ of total deaths. The age-adjusted death rate is 306.02 per 100,000 population, which puts Kazakhstan as eighth in the world, and approximately 9.6-fold higher than Japan (2018: 31.55 per 100,000 of population ranks Japan \#181 in the world) and 4.05-fold higher than Austria (a death rate of 75.74 per 100,000 population ranks Austria \#143 in the world) [1].

Kazakhstan is located in the middle of Central Asia on the ancient Great Silk roads. Its vast territory covers 2,724,900 $\mathrm{km}^{2}$ and it is the world's 9th largest country. Kazakhstan has a population of more than 17 million people (2016), with 131 ethnicities, including Kazakh (63\% of the population), Russian, Ukrainian, German, Uzbek, Tatar, and Uyghur as the most predominant groups. Historically, the ethnic Kazakhs were nomadic and migration led to the admixing of western and eastern tribes. Kazakhstan is ethnically and culturally diverse, which is due, in part, to the forced migration of settlers and mass deportations of ethnic groups, starting in the 19th century until the first third of the 20th century [2]

Genetic studies in Kazakhstan are challenging because of the genetic heterogenicity introduced by many ethnicities. However, data from diverse, heterogeneous populations exposed to the same environmental conditions and similar lifestyles yield important information on natural genetic plasticity. Such data are fundamental to genetic epidemiology and are critical to dissect natural polymorphisms from pathogenic alterations [3]. Genome-wide data and linkage disequilibrium patterns are unavailable for Central Asian populations and are not represented in publicly available databases.

Cardiovascular disease encompasses a range of conditions from diseases of the vasculature, myocardial infarction, and congenital heart disease, most of which are heritable. Enormous effort has been invested in understanding the genes and specific DNA sequence variants responsible for this heritability $[4,5]$.

Dilated cardiomyopathy (DCM) accounts for $30-40 \%$ of all heart failure cases and is a leading cause of heart transplantation [6]. An autosomal dominant inheritance pattern of transmission and some autosomal recessive or X-linked recessive familial cases have been reported as indicated by the familial aggregation of DCM (30-50\% of all DCM cases) $[5,6]$. Mutations in more than 30 disease genes have been linked as causative mechanisms of DCM [6,7]. In contrast, $\mathrm{CHD}$, which is typically a result of coronary artery disease (CAD), is a complex disease driven by interactions between genetic factors and environmental stimuli and stressors. The genetic basis of CAD/CHD has been addressed by multiple genome-wide association studies (GWAS) enrolling thousands of individuals [8-11]. Genomic risk scores (GRS) have been developed using millions of datapoints (SNPs) and plasma markers. These can contribute to our 
113 understanding of critical mechanisms to provide the maximum benefit to the individual, 114 especially before the early stages of pathogenesis. Classical CHD markers defined by the 115 Framingham risk score (FRS), including age, cholesterol, smoking status, blood-pressure, and 116 diabetes status are not predictive in a timely way [3]. Genetic risk loci have been reported for cell 117 proliferation genes, inflammation and immunity related genes, cholesterol and lipid biogenesis

118 genes, among others. However, only a relatively minor risk could unambiguously be attributed to the wealth of common genetic variants in CHD heritability. Family-based analyses revealed different heritability estimates for distinct sub-phenotypes of CHD [12]. There is a remarkable consistency in genetic association findings across cohorts (with varying phenotype definitions), underscoring that different manifestations of CHD may have a common genetic architecture $[8,13]$. Rare variants with a larger impact and/or more common variants with a smaller biological impact were proposed to cause the observed missing heritability $[6,14,15]$. Ventricular arrhythmias in patients with structural heart disease are responsible for the majority of sudden cardiac deaths (SCD). CAD, previous myocardial infarction is the most common heart disease in which sustained ventricular tachycardia (VT) occurs and reentry is the predominant mechanism. Other cardiac conditions, such as idiopathic DCM, Chagas disease, sarcoidosis, arrhythmogenic cardiomyopathies, and repaired congenital heart disease may also present with VT in follow-up [16]. Recurrent ventricular tachycardia (VT) is an important cause of increased morbidity and mortality in patients with non-ischemic DCM. DCM differs from postinfarction ischemic cardiomyopathy by comprising multiple different etiologies with variable disease progression and prognosis. There is a need for an individualized approach to risk stratification and treatment based on genetic information. Idiopathic ventricular tachycardia is defined as VT that occurs in patients without structural heart disease, metabolic abnormalities, or long QT syndrome. 10\% of all patients referred for evaluation of VT show no obvious structural heart disease. Idiopathic VT is characterized by a structurally normal heart and QRS morphology consistent with site of origin from typical locations of idiopathic ventricular arrhythmias (in particular, the ventricular outflow region). An absence of structural heart disease is usually suggested if an electrocardiogram (ECG) (except in Brugada syndrome and long QT syndrome [LQTS]), echocardiogram, and coronary arteriogram are collectively normal [17]. However, magnetic resonance imaging (MRI) may identify structural abnormalities even if all other test results are normal. Idiopathic VT comprises multiple discrete subtypes that are differentiated by their mechanism, QRS morphology, site of origin, the response to pharmacologic agents, and evidence of catecholamine dependence. They include right ventricular (RV) monomorphic extrasystoles, RV outflow tract (RVOT) VT, left ventricular (LV) outflow tract (LVOT) VT, idiopathic LV tachycardia (ILVT), and idiopathic propranolol-sensitive (automatic) VT (IPVT). Idiopathic VT from the RVOT and LV 151 are monomorphic and generally not familial. Catecholaminergic polymorphic VT (CPVT), Brugada syndrome, and LQTS are inherited ion channelopathies [17]. 
152 Polymorphic VT may cause syncope and sudden death in Brugada syndrome. Patients with 153 idiopathic VT monomorphic forms have a better prognosis than do patients with polymorphic

154 VT and structural heart disease. Prognosis for patients with VT secondary to ion channelopathies 155 is variable [17].

15692 patients diagnosed with ventricular tachycardia (VT) with either coronary heart disease 157 (CHD), dilated cardiomyopathy (DCM) or idiopathic ventricular tachycardia (iVT), were 158 enrolled in a study to evaluate the genetic profile and variants in known cardiac risk genes by 159 targeted next generation sequencing (NGS). We hypothesized that shared morphological and 160 phenotypical features among these subgroups might originate from an overlapping molecular 161 basis. In addition, we assumed that the spacious genepool of the population of Kazakhstan that is 162 fueled by more than 100 different ethnic groups deems this study cohort a challenging but 163 valuable source for interpreting disease-associated genetic variations. Our results provide an 164 165 important contribution to the understanding of human genetic diversity.

\section{Materials \& Methods}

\section{Study population}

Studies were performed in accordance with the institutional guidelines for human research and the principles of the Declaration of Helsinki. Our research protocol was reviewed and approved by the Ethics Committee of the Center for Life Sciences, National Laboratory Astana, Nazarbayev University and Ethics Committee of the National Research Cardiac Surgery Center (NRCSC), Astana (\#20-20/09/17). Written informed consent and permission to publish data was obtained from all research subjects (or their parents for children under 16 years old).

Patients with ventricular tachycardia were enrolled during 2014-2016 at the NRCSC, Astana, Kazakhstan. The study cohort consisted of 92 unrelated patients with ventricular tachycardia (VT) and different background conditions: DCM (DCM VT, n=32), CHD (CHD VT, n=23), and idiopathic VT (iVT, $n=37$ ). A clinical diagnosis of patients was verified in all patients by the authors (M.B., O.N., G.R, G.K.) and further experienced cardiologists of the NRCSC, according to international guidelines and criteria (Supplemental file 1). Patient characteristics and functional parameters are summarized in Table 1 and the statistical testing of clinical parameters is in Table 2. Detailed information on each patient characteristic available in Supplemental file 2: Table S1.

Patients were from Kazakhstan, with Asian and/or Caucasian ancestry. The cohort included sporadic (65/92) and familial (25/92) cases as well as two cases with unknown familial history. The mean age at the time of initial evaluation and diagnosis of the CHD patients was $62.3 \pm 8.7 \mathrm{y}$ ( $95 \%$ male), $43 \pm 13.3 y$ ( $65.6 \%$ male) for the DCM subgroup, and $37.1 \pm 19.2 \mathrm{y}(43.2 \%$ male) for the iVT sub-group.

We used sequence data from 60 unrelated Kazakh individuals without known CHD, DCM, and iVT as a comparison group called the Kazakh control group (KCG) representing the general 
192 population. The KCG average age was $37.5 \pm 10.9$ years, and the ratio of male:female was

193

194

195

196

197

198

199

200

201

202

203

204

205

206

207

208

209

210

211

212

213

214

215

216

217

218

219

220

221

222

223

224

225

226

227

228

229

230

231 0.63:0.37, respectively. We deposited sequence data from the Kazakh control group in a publicly accessible repository (Submission ID: SUB7590848, BioProject ID: PRJNA646320). Our data are registered with the BioProject database (http://www.ncbi.nlm.nih.gov/bioproject/646320; BioProject ID PRJNA646320).

\section{Design of the target region for gene enrichment}

We designed a custom targeted gene panel using HaloPlex Target Enrichment technology with Agilent Technologies SureDesign software (https://earray.chem.agilent.com/suredesign/). This system allowed us to simultaneously sequence 96 known diagnostic genes for cardiac cardiomyopathies and arrhythmias and additional loci associated with cardiac disorders. HaloPlex technology uses custom molecular inversion probes (SureDesign software, Agilent) for selective circularization-based target enrichment. The diagnostic genes were compiled for 96 genes and target regions that are known causes or candidate genes for cardiac cardiomyopathies and arrhythmias from PubMed and clinical variant databases (such as HGMD and ClinVar) (Supplemental file 3). The candidate gene library design covers a total target region of 463.767 kbp (which was used as input for eArray (Agilent Technologies, Santa Clara, California, USA) to design the custom capture-oligonucleotides for in-solution target enrichment with 406.062 analyzable target bases. The analyzable target bases (ATB) included all exonic and proximal intronic (+/-10bp) sequence information for the 96 cardiac risk genes. ATB are represented by 2,017 target loci.

\section{Target DNA enrichment and next-generation sequencing}

DNA was isolated from fresh-frozen EDTA-blood samples of the patients and processed according to the standard HaloPlex Target Enrichment System Protocol (version D.5, May 2013, Agilent Technologies, Santa Clara, CA, US) using the standard HaloPlex 96 indexing primer cassette. We used the SureSelect Target Enrichment System (Agilent Technologies, Santa Clara, CA, US) for capturing the designed regions. All libraries were quality checked on a 2100 BioAnalyzer (Agilent Technologies, Santa Clara, CA, US) using the High Sensitivity DNA Assay kit, pooled at equimolar amounts and sequenced on a HiSeq2000 platform using 2x150bp paired-end standard sequencing.

\section{Sequencing data processing and variant annotation}

Sequence data processing and variant calling was conducted using Agilent NGS data analysis software SureCall version 2.0.7.0 (Agilent Technologies, Santa Clara, CA, USA) with standard 
232

233

234

235

236

237

238

239

240

241

242

243

244

245

246

247

248

249

250

251

252

253

254

255

256

257

258

259

260

261

262

263

264

265

266

267

268

269

270

271

settings of the HaloPlex pipeline. Resulting variants were further matched with entries in the Human Gene Mutation Database (HGMD [18]) and annotated with ANNOVAR [19]. We included the predictions from the database of human non-synonymous SNVs dbNSFP [20] to achieve better scoring. The clinical significance of the sequence variants was interpreted according to the guideline developed by the American College of Medical Genetics and Genomics (ACMG) and the Association for Molecular Pathology (AMP) in 2015 [21]. The ClinVar [22] and Varsome [23] databases were applied for the variant classification.

The KCG sequencing project identified a total of 2,150 genetic variants in 60 individuals, with a mean coverage of 157 -fold at the ATB. The mean coverage of the 92 samples of the cardiology study cohort at the target loci was 707.62-fold and revealed 2,403 distinct genetic variants in the 92 patients. We first eliminated the known common variants with frequencies above $0.5 \%$ using a stepwise approach in commonly referenced databases like the ESP6500 or the $=00 \mathrm{Genomes}$ $\mathrm{Db}$ or SNPDb130. All synonymous variants and variants observed in the KCG were subtracted from the patient cohort data set, yielding a total of 337 individual non-synonymous variants. The resulting data set, after manual curation, contained 307 individual variants for the overall study population.

\section{In silico prediction analysis and pathogenicity inference}

The pathogenic potential of each variant (HGMD-listed variants and novel or rare variants) was predicted using a combined score from 10 prediction tools: SIFT_score/pred, Polyphen2_HDIVscore/pred, Polyphen2_HVAR_score/pred, LRT_score/pred, MutationTaster_score/pred, MutationAssessor_score/pred, FATHMM_score/pred, RadialSVM_score/pred, LR_score/pred, and MetaSVM_score/pred. Class I (highest pathogenic potential) variants were predicted as being disease-causing by at least 7 of the tools; class II (intermediate pathogenic potential) variants were predicted as being disease-causing by 4-6 of the tools; class III (low pathogenic potential) were predicted as being disease-causing by 1-3 prediction tools; and class IV (benign) was predicted as being disease-causing by none of the tools (0). The PhyloP100 and SiPhy_29 scores are conservation scores and are not designed specifically for finding causal variants for Mendelian diseases, but for finding functionally important sites. Variants that confer increased susceptibility may be scored well. Polyphen2hdiv is commonly used when evaluating rare alleles at loci that are potentially involved in complex phenotypes, dense mapping of regions identified by genome-wide association studies, and analysis of natural selection from sequence data. For further in silico analysis, sequence variants were interpreted according to the ACMG/AMP classifications: "pathogenic," "likely pathogenic," "uncertain significance," "likely benign," and "benign". The two sets of criteria were for the classification of pathogenic or likely pathogenic variants and for the classification of benign or likely benign variants [21]. Each pathogenic criterion was weighted as very strong (PVS1), strong (PS1-4), moderate (PM1-6), or supporting (PP1-5), and each benign criterion was weighted as stand-alone (BA1), strong (BS1-4), or supporting (BP1-6). The ClinVar 
272 (http://www.ncbi.nlm.nih.gov/clinvar) database was used for its clinical assertions and evidence

273 for the variant classification. Gene symbols recognized by ClinVar were entered and we obtained

274 results with variations affecting the genes. We used the search engine Varsome

275 (https://varsome.com/), which has information from 30 external databases, to look up variant

276 pathogenicity. Pathogenicity of the identified sequence variants is reported using an automatic

277 variant classifier that evaluates the submitted variant according to the ACMG guidelines [21],

278 classifying it as one of 'pathogenic', 'likely pathogenic', 'likely benign', 'benign' or 'uncertain'

279 significance. We summarized the information about the HGMD-listed variants and the

280 pathogenicity found in the ClinVar, Varsome and final verdict according to the ACMG/AMP

281 guidelines in Table S2 (Supplemental file 4: Table S2).

282

283

284

285

286

287

288

289

290

291

292

293

294

295

296

297

298

299

300

301

302

303

304

305

306

307

308

309

310

311 The clinical characteristics of the patients in the three clinical subgroups are summarized in

312 Table 1 and listed in detail in Supplemental file: Table S1. The predominant NYHA functional

Peer) reviewing PDF | (2020:03:47033:2:0:NEW 26 Nov 2020) 
313 classes were II-III for CHD, III-IV for DCM, and I-II for the iVT subgroup. LVEF, LA, LV 314 ESD, and LV EDD significantly differed between the iVT and the two other subgroups (Table

315 2). The proportion of familial cases was nearly the same in all three subgroups, ranging from 25 $31629.7 \%$.

317

\section{Genetic variants}

319

320

321

322

323

324

325

326

327

328

329

330

331

332

333

334

335

336

337

338

339

340

341

342

343

344

345

346

347

348

349

350

351

352

Targeted enrichment and sequencing, variant calling and stepwise filtering of the annotated variants identified a total of 307 unique variants in 74 genes totaling up in 456 variants for the overall study group. The frequency and pathogenicity of variants associated with the different arrhythmogenic syndromes within the different subgroups studied is shown in (Supplemetal file 4: Table S2). Variants included: one in/del variant, four splice-site variants, and 451 singlenucleotide variants (SNV) within the coding exonic regions. Seven $(0.15 \%)$ of the SNVs were unique stop-gain variants, three of which were residing in the TTN gene. 168 HGMD mutations (61 unique) were observed in 37 genes. $33 \%$ of the HGMD mutations were predicted to have an increased pathogenic potential (class I and II variants); 49\% were classified class III variants and $18 \%$ of the observed HGMD variants were classified as benign by all prediction algorithms (Fig. 1). In contrast to the HGMD variants, a higher proportion (40\%) of the novel and rare variants $(\leq 0.5 \%$ in ESP 6500 or $1000 \mathrm{G} \mathrm{db}$ ) was predicted to be pathogenicity classes I and II (Fig. 1). Variants with the highest pathogenicity score (class I variants) made up approximately $8.7 \%$ of the CHD VT subgroup, and $6.3 \%$ and $18.9 \%$ in the DCM VT and the iVT groups, respectively (Fig. 2). The prevalence of class II variants was moderately lower (30.4\%) in the CHD VT group for the DCM VT and the iVT groups (31.4\% and $43.2 \%$, respectively).

Statistical testing of and/or rare variants between the different clinical subgroups (testing was done including and omitting titin variants) indicated no difference in the average number of HGMD variants or rare variants alone between the three subgroups (Supplemental file 5: Table S3, Supplemental file 6:Fig. S1a, Fig. S1b).

Classification of 307 variants according to ACMG guidelines showed that $9(2.9 \%)$ variants were classified as pathogenic, $9(2.9 \%)$ were likely pathogenic, 98 (31.9\%) had uncertain significance, $73(23.8 \%)$ were likely benign and 118 (38.4\%) were benign (Fig.3, Supplemental file 4: Table S2). ACMG pathogenic and likely pathogenic variants were observed in classes III and IV, and contrary benign and likely benign variants were observed in class I and class II variants. Most variants were variants of uncertain significance.

\section{Measure of molecular burden}

We used the cumulative potential pathogenic variance to determine whether there was a difference in the genetic variation per patient, as a measure of the molecular burden, within the three different subgroups (Table 3). 10 patients with CHD VT (43.5\%) carried at least one class I variant, averaging 1.3 class I variants per positive individual. The same variant frequency was

Peer) reviewing PDF | (2020:03:47033:2:0:NEW 26 Nov 2020) 
353

354

355

356

357

358

359

360

361

362

363

364

365

366

367

368

369

370

371

372

373

374

375

376

377

378

379

380

381

382

383

384

385

386

387

388

389

390

391

392

observed for the DCM VT and the iVT subgroups (31.3\%; 10 individuals carrying 13 class I variants and 20 individuals carrying 26 class I variants, respectively). 69.6\% (CHD VT), 75\% (DCM VT), and $81 \%$ (iVT) of the patients carried about two class I or II (intermediate pathogenic potential) variants. The inclusion of class III variants (low pathogenic potential) increased the average variant frequency to 4.05 (CHD VT; $95.7 \%$ of patients), 4.23 (DCM VT; 96.9\%), and 4.44 (iVT; 97.3\%) per patient (Table 3, Fig. S2).

None of the CHD VT patients and only one DCM VT and iVT patient carried zero HGMD or other rare class I-IV variants (Supplemental file 8: Table S4). More than 75\% (CHD VT: 86.9\%, DCM VT: $78.13 \%$, iVT: $81 \%$ ) of the patients carried at least one HGMD mutation, irrespective of the disease group.

\section{Distribution of the functional effects of detected mutations}

We evaluated whether there might be a differential distribution of variants between the disease groups in relation to their functional context. We grouped the genes into seven categories using information from GeneCards ${ }^{\circledR}$ and The Human Gene Database https://www.genecards.org/ (cell membrane, cytoskeleton, sarcomere, metabolism, intercalated disc, ion flux, and nucleus) based on their molecular function and/or subcellular association. Distribution of class I-IV variants according to their molecular function/association are shown in Table 4 . There was a moderate, but statistically insignificant, underrepresentation of variants in the cell membrane (4.3\%) genes and intercalated disc (4.3\%) genes in CHD VT patients compared to the other groups (DCM VT: 9.4 and 12.5 ; iVT: $10.8 \%$ and $21.6 \%$, respectively) when we included only class I and II variants in the analysis (Fig. 4 and Supplemental file 9, Fig. S3 and Supplemental file 10: Table S5). Variants in the metabolism-associated genes were moderately overrepresented in the CHD VT subgroup (13\%) compared to the DCM VT subgroup (3.1\%) alone.

Based on their relative frequencies HGMD mutations in LAMA2 (34.3\%), MYBPC3 (31.2\%), MYH6 (18.7\%), KCNQ1 (15.6\%), GAA (15.6\%) and DSG2 (12.5\%) were predominant in the DCM VT subgroup (Fig. 5). The mutation and variant distribution of the CHD VT subgroup strongly overlapped with the patterns for the other subgroups (Supplemental file 4: Table S2, Supplemental file 11: Table S6, and Supplemental file 12: Fig. S4). PRKAG2 mutations p.G100S $(\mathrm{n}=3,13 \%, \mathrm{CM} 136115)$ and novel p.H222Q variant were observed in four CHD VT patients (Supplemental file 4: Table S2). Statistical testing suggested a trend towards an increased frequency of PRKAG2 variants in the CHD subgroup (CHD VT: $13.04 \%$ vs. DCM VT: $3.1 \%$ and iVT: 2.7\%; p-value 0.053) (Supplemental file 12, Fig. S4). There was a prevalence of iVT mutations in genes encoding ion flux.

There were 9 pathogenic variants of ACMG, including W746C in GAA in patients with CHD, R218Q KCNJ2 and R5338X TTN in patients with iVT, and F244L MYH7, Q353X LMNA, L17465X TTN, W21011X TTN, c.2334+1G>A DSG2, c.477+1G $>\mathrm{A} K C N Q 1$ in patients with DCM (Supplemental file 4: Table S2). Sanger sequencing confirmed some of the observed genetic variants. (Supplemental file 13: Figure S5). 
393

394

395

396

397

398

399

400

401

402

403

404

405

406

407

408

409

410

411

412

413

414

415

416

417

418

419

420

421

422

423

424

425

426

427

428

429

430

431

432

\section{Genetic variants in the healthy Kazakh group}

From a total of 2,150 variants, observed in the practically healthy individuals, $\mathrm{KCG}(\mathrm{n}=60), 475$ were common polymorphisms and thus subtracted from further analysis. The remaining 1,675 variants included 68 exonic variants ( 37 synonymous, three frameshift deletions, three in/del non-frameshift deletions, and 25 non-synonymous single nucleotide variants). Variants with a MAF (minor allele frequency) of $\geq 0.5 \%$ in the ESP 6500 or the $1000 \mathrm{G}$ yielded 58 exonic variants were also excluded. $3(5.2 \%)$ were predicted to be class I, $11(19 \%)$ were class II, $36(62 \%)$ were class III, and $8(13.8 \%)$ were predicted to be class IV. Thus, the average frequency of a class I variant in the $\mathrm{KCG}(\mathrm{n}=60)$ was $5 \%$. We analyzed the presence and frequency of 307 genetic variants found in patients in the KCG group. 58 genetic variants were observed in KCG and 5 of these genetic variants was a mutant minor allele (Supplemental file 4: Table S2).

\section{Discussion}

We evaluated the contribution of molecular genetic variants in genes associated with cardiac disorders in Kazakhstani population. We identified a significant proportion of possible pathogenic variants using molecular genetic screening with a targeted next-generation sequencing (NGS) panel [24,25]. We obtained data on the distribution of genetic variants, the number of mutations, and the mutational burden of patients with ventricular tachycardia of various etiology.

NGS technologies have emerged as an efficient alternative to Sanger-sequencing, providing the analytical characteristics for the comprehensive exploration of genetic mechanisms [26,27]. Furthermore, it is believed that NGS will be increasingly important in the studies of monogenic and complex diseases, such as common cardiovascular diseases (CAD, cardiomyopathies and others) in which one or more variants in a single gene, or multiple variants in different genes, are involved $[6,7,26,27]$. The ability of NGS to generate high-throughput qualitative and quantitative sequence information has enabled investigations that were previously technically infeasible or cost prohibitive [28].

There are some disadvantages to the use of NGS, including the incomplete representation and coverage of exons, which poses the risk of limiting sensitivity and the inability to detect clinically significant mutations. Targeted enrichment of certain genes followed by the use of NGS for high-throughput genetic testing of genes for heart disorders is now becoming feasible and technically proven. This has been evidenced by the almost complete coverage and high accuracy of the approach, offering greater sequencing depth with reduced costs and data burden $[7,28]$.

We sequenced three groups of patients with CHD VT, DCM VT and VT of unknown etiology (idiopathic) in our study to identify genetic variants that were associated with the 3

cardiovascular phenotypes and to evaluate the level of genetic variation in cardiac risk genes in

Peer) reviewing PDF | (2020:03:47033:2:0:NEW 26 Nov 2020) 
433 these distinct subgroups. We designed and optimized a custom target-enrichment assay of 96

434 genes associated with cardiac disorders using Haloplex technology (Agilent Technologies, Santa 435 Clara, USA) [24,25].

436 Targeted enrichment and sequencing and stepwise filtering of the annotated variants identified a 437 total of 307 unique variants in 74 genes totaling up in 456 variants for the overall study group.

438 The filtering step is crucial for bioinformatics analysis to reduce the number of probable and 439 potentially pathogenic variants such as the exclusion of common variants present in the Single 440 Nucleotide Polymorphism database (dbSNP). Filtering is based on assumptions about the

441

442

443

444

445

446

447

448

449

450

451

452

453

454

455

456

457

458

459

460

461

462

463

464

465

466

467

468

469

470

471

472 attributes of the disease-causing variant(s), including the effect of the variant on the protein, the presumed absence of the variant in the dbSNP database, or the frequency cutoffs based on minor allele frequency from the 1000 Genomes Project [28].

The DCM and other common congenital heart disorders affect approximately 1-4 people per 10,000 population. We selected a MAF cut-off of $0.5 \%$ for rare variants in order to balance the rate of false positives (non-pathogenic variants) at the cost of losing variants with

moderate/intermediate pathogenicity (true positives) that provoke increased susceptibility for complex diseases like CHD. With the introduction of multigene panels, exome sequencing, and whole-genome sequencing, the numbers of variants identified per person has increased progressively, and disease mutation databases contain potentially benign variants that were previously classified as disease causing. The interpretation of genetic variants is complex.

We used a combined score of 10 prediction tools to determine the pathogenic potential of each variant (HGMD listed variants and novel or rare variants). Class I (highest pathogenic potential) variants were predicted as being disease causing by at least 7 of the tools; class II (intermediate pathogenic potential) variants were predicted as being disease causing by 4-6 of the tools; class III (low pathogenic potential) were predicted as being disease causing by 1-3 prediction tools; and class IV (benign) was predicted as being disease causing by none of the tools ( 0$)$.

As expected, the MAF was the lowest for the variants with the most severe pathogenicity (class I) (0.000279), followed by intermediate pathogenicity variants (class II) with an MAF of 0.00267 being nearly a-10-fold higher than that for class I. The average MAF for the variants with the lowest pathogenicity (class III variants with low pathogenic potential) was relatively common at 0.00372 .

The pathogenicity of sequence variants was classified using an automatic variant classifier according to the ACMG guidelines. The classifications were: 'pathogenic', 'likely pathogenic', 'likely benign', 'benign', or 'uncertain significance'. Information about HGMD listed variants and the classification of pathogenicity found in the ClinVar, Varsome and final verdict according to the ACMG/AMP were summarized in Table S2 (Supplemental file 4: Table S2).

We classified 307 variants according to ACMG guidelines, which showed that $9(2.9 \%)$ variants were pathogenic, $9(2.9 \%)$ were likely pathogenic, $98(31.9 \%)$ had uncertain significance, 73 (23.8\%) were likely benign and 118 (38.4\%) were benign (Fig.3). ACMG pathogenic and likely pathogenic variants were observed in classes III and IV, and contrary benign and likely benign variants were observed in class I and class II variants. Most variants had uncertain significance. 
473 Our results show that complex methods are required to make a final interpretation of sequenced 474 variants.

475 Patients of all subgroups were clinically diagnosed according to common international 476 classification criteria. Despite the many common genetic risk variants identified for CHD in 477 GWAS, they only account for a small percentage of the expected heritability $[27,29]$. The 478 predisposition to CHD is estimated to be approximately 50\% genetic, although the 36 variants

479

480

481

482

483

484

485

486

487

488

489

490

491

492

493

494

495

496

497

498

499

500

501

502

503

504

505

506

507

508

509

510

511

512 identified by CARDIoGRAM and the follow-up CARDIoGRAMplusC4D project only accounted for about $10 \%$ of the heritability. Rare risk variants with minor allele frequencies $\leq 1$ $5 \%$, complex gene-gene interactions (epistasis), and undiscovered common variants are thought to cause this discrepancy. We sequenced 23 individuals with CHD VT and observed a spectrum of genetic variation that quantitatively (frequency of genetic variants) and qualitatively (molecular function) strongly overlapped with DCM VT and iVT. 43.5\% (10/23) of CHD VT patients carried a class I variant, whereas only 5\% (3/60) of the control cohort (KCG), 31.3\% (13/32) of the DCM VT and 54.1\% (20/37) of iVT patients carried a class I variant. If class II variants were added, $69.5 \%$ (16/23) of CHD VT, 75\% (24/32) of DCM VT and 81.1\% (30/37) of iVT patients carried on average two variants of high to intermediate pathogenicity. High- to intermediate pathogenicity variants in $L A M A 2, M Y B P C 3, M Y H 6, K C N Q 1, G A A$, and DSG2 predominated in CHD VT patients at similar frequencies as those observed for DCM VT and iVT patients. This similarity points to a common molecular disease-association. Independent of multiple tested disease-associated mutations (HGMD) and rare or newly identified variations with increased pathogenic potential, there was no statistically significant difference in the frequency of genetic variation between the three subgroups. Our results confirmed that DCM and iVT patients frequently carry multiple mutations or variants with high pathogenic potential, which has been shown in previous research. The high frequency of rare genetic variants with increased pathogenic potential (class I and II) in CHD patients was unexpected. The commonly referenced concepts of cardiomyopathies as monogenic disorders has been challenged [6,29, 30], indicating complex interactions of genes and the significance of rare variants. Distinct clinical phenotypes, including LQT, Brugada syndrome and HCM, revealed that multiple mutations, and rare potential pathogenic and functional variants in affected individuals could synergistically or additively alter penetrance, age-of-onset, or disease progression [7, 31-33]. TTN, GAA, LAMA2 and MYBPC3 harbored the most variants in the three subgroups which confirm the high impact of these genes in complex pathogenesis of cardiomyopathies and VT demonstrated in previous studies [34, 35, 36, 37].

Classification of the variants according to their cellular function showed that sarcomere function, ion-flux, nuclear function, and metabolism were predominantly affected by variants with the highest pathogenic potential (class I variants) in CHD VT patients. A similar pattern was observed for class I variants in DCM VT and iVT patients. In addition, iVT patients carried variations potentially affecting the cytoskeleton and intercalated disc. Pooling class I-III variants yields metabolism-associated variants in $>60 \%$ of CHD VT patients, which is second behind variants in sarcomere genes. On a gene basis, PRKAG2 mutations were overrepresented in the 
513 CHD sub-group versus DCM and iVT, (p-values $=0.053$ and 0.054 , respectively). The mean age

514 of the four heterozygous mutation carriers was 67.7 years (+/- 5.1y). PRKAG2 encodes the $\gamma 2$

515 regulatory subunit of the AMP-activated protein kinase AMPK and mutation-associated defects

516 account for a cardiac syndrome triad consisting of familial ventricular preexcitation [38],

517 conduction system disease, and cardiac hypertrophy mimicking (HCM) [39], with a significant

518 proportion of those progressing to DCM. HCM-associated PRKAG2 mutations are generally not

519 associated with myocyte and myofibrillar disarray, which are the pathognomonic features of

$520 \mathrm{HCM}$, but with pronounced vacuole formation within myocytes due to excessive glycogen

521 accumulation [40,41]. This may be explained by the central regulatory function of AMPK during

522 acute low-energy states in which ATP-consuming pathways are shut off, like glycogen,

523 cholesterol and fatty acid synthesis and the ATP-producing pathways are enhanced, such as fatty

524 acid oxidation and glucose uptake. The potential functional role of AMPK in atherosclerosis has

525 recently been shown by the protective effect of melatonin on the cardiovascular system, since

526 flow shear stress-induced apoptosis in bone marrow mesenchymal stem cells could be reversed

527 via the activation of AMPK [42].

528

529

Conclusions

530

531 We showed that in patients with VT, secondary to coronary artery disease, DCM, and idiopathic

532 etiology, multiple rare mutations and clinically significant sequence variants in classic cardiac

533 risk genes associated with cardiac channelopathies and cardiomyopathies were found in a similar

534 pattern and at a comparable frequency. CHD VT patients were found to carry rare genetic

535 variants with an increased pathogenic potential at a comparable frequency as DCM VT and iVT

536 patients. These variants were found in genes related to sarcomere function, nuclear function, ion

537 flux, and metabolism. Our study size was limited but this pilot study suggests that monogenic

538 diseases can serve as an insightful model for complex disorders. Patients with coronary heart

539 disease, dilated cardiomyopathy, and idiopathic ventricular tachycardia share overlapping

540 patterns of pathogenic variation in cardiac risk genes. A greater in-depth statistical analysis like

541 sub-grouping of participants according to other features like ethnicity, severity, or anamnesis

542 (familial vs. sporadic, etc.) was not possible at this stage due to the group size limitation.

543 Additional studies including more patients with and without ventricular tachycardia will be

544 needed to generate a deeper insight into genotype-phenotype correlation of CHD and

545 cardiomyopathies and between idiopathic ventricular tachycardia types.

546

\section{Acknowledgements}

548 We thank A. Groselj-Strele at the Core Facility Computational Bioanalytics (Med. Univ. of

549 Graz) for statistical analysis.

550 


\section{References}

552 1. Data Source: World Health Organization 2018. World health rankings

553

554

555

556

557

558

559

560

561

562

563

564

565

566

567

568

569

570

571

572

573

574

575

576

577

578

579

580

581

582

583

584

585

586

587

588

589

590

https://www.worldlifeexpectancy.com/kazakhstan-coronary-heart-disease

2. Lee JH., Akilzhanova A. Kazakhstan. In: Colditz GA, editor. The SAGE Encyclopedia of Cancer and Society. Thousand Oaks: SAGE Publications, Inc.; 2015. p646-647. DOI: http://dx.doi.org/10.4135/9781483345758.n313.

3. Akilzhanova A, Guelly C, Nuralinov O, Nurkina Z, Nazhat D, Smagulov S, Tursunbekov A, Alzhanova A, Rashbayeva G, Abdrakhmanov A,Dosmagambet S, Trajanoski S, Zhumadilov Z, Sharman A, Bekbosynova M. RYR2 sequencing reveals novel missense mutations in a Kazakh idiopathic ventricular tachycardia study cohort. PLoS One 2014;9(6):e101059.

4. Abraham G, Bhalala OG, de Bakker PI, Ripatti S, Inouye M. Towards a molecular systems model of coronary artery disease. Curr Cardiol Rep 2014;16(6):488.

5. Al-Hassnan ZN, Shinwari ZM, Wakil SM, Tulbah S, Mohammed S, Rahbeeni Z, Alghamdi M, Rababh M, Colak D, Kaya N, Al-Fayyadh M, Alburaiki J. A substitution mutation in cardiac ubiquitin ligase, FBXO32, is associated with an autosomal recessive form of dilated cardiomyopathy. BMC Med Gen 2016;17:3.

6. Haas J, Frese KS, Peil B, Kloos W, Keller A, Nietsch R, Feng Z, Müller S, Kayvanpour E, Vogel B, Sedaghat-Hamedani F, Lim WK, Zhao X,Fradkin D, Köhler D, Fischer S, Franke J, Marquart S, Barb I, Li DT, Amr A, Ehlermann P, Mereles D, Weis T, Hassel S, Kremer A, King V, Wirsz E, Isnard R, Komajda M, Serio A, Grasso M, Syrris P, Wicks E, Plagnol V, Lopes L, Gadgaard T, Eiskjær H, Jørgensen M, Garcia-Giustiniani D, OrtizGenga M, Crespo-Leiro MG, Deprez RH, Christiaans I, van Rijsingen IA, Wilde AA, Waldenstrom A, Bolognesi M,Bellazzi R, Mörner S, Bermejo JL, Monserrat L, Villard E, Mogensen J, Pinto YM, Charron P, Elliott P, Arbustini E, Katus HA, Meder B. Atlas of the clinical genetics of human dilated cardiomyopathy. Eur Heart J 2015;36(18):1123-1135a.

7. Meder B, Haas J, Keller A, Heid C, Just S, Borries A, Boisguerin V, ScharfenbergerSchmeer M, Stähler P, Beier M, Weichenhan D, Strom TM, Pfeufer A,Korn B, Katus HA, Rottbauer W. Targeted next-generation sequencing for the molecular genetic diagnostics of cardiomyopathies. Circ Cardiovasc Gen 2011;4(2):110-122.

8. Lieb W, Vasan RS. Genetics of coronary artery disease. Circulation. 2013;128(10):11311138.

9. CARDIoGRAMplusC4D Consortium. Large-scale association analysis identifies new risk loci for coronary artery disease. Nat Genet 2013;45(1):25-33.

10. CARDIoGRAM Consortium. Design of the coronary artery disease genome-wide replication and meta-analysis (cardiogram) study: A genome-wide association meta-analysis involving more than 22000 cases and 60000 controls. Circ Cardiovasc Genet 2010;3(5):475-483.

11. Coronary Artery Disease (C4D) Genetics Consortium. A genome-wide association study in Europeans and South Asians identifies five new loci for coronary artery disease. Nat Genet 2011;43(4):339-344. 
591

592

593

594

595

596

597

598

599

600

601

602

603

604

605

606

607

608

609

610

611

612

613

614

615

616

617

618

619

620

621

622

623

624

625

626

627

628

629

630

631

12. Fischer M, Broeckel U, Holmer S, Baessler A, Hengstenberg C, Mayer B, Erdmann J, Klein G, Riegger G, Jacob HJ, Schunkert H. Distinct heritable patterns of angiographic coronary artery disease in families with myocardial infarction. Circulation 2005;111(7):855-862.

13. Kitsios GD, Dahabreh IJ, Trikalinos TA, Schmid CH, Huggins GS, Kent DM. Heterogeneity of the phenotypic definition of coronary artery disease and its impact on genetic association studies. Circ Cardiovasc Genet 2011;4(1):58-67.

14. Schunkert H, Erdmann J, Samani NJ. Genetics of myocardial infarction: A progress report. Eur Heart J 2010;31(8):918-925.

15. Manolio TA, Collins FS, Cox NJ, Goldstein DB, Hindorff LA, Hunter DJ, McCarthy MI, Ramos EM, Cardon LR, Chakravarti A, Cho JH, Guttmacher AE, Kong A, Kruglyak L, Mardis E, Rotimi CN, Slatkin M, Valle D, Whittemore AS, Boehnke M, Clark AG, Eichler EE, Gibson G, Haines JL, Mackay TF, McCarroll SA,Visscher PM. Finding the missing heritability of complex diseases. Nature 2009;461(7265):747-753.

16. Hadid C. Sustained ventricular tachycardia in structural heart disease. Cardiol J. 2015;22(1):12-24. doi:105603/CJ.a2014.0069.

17. Srivathsan K, Lester SJ, Appleton CP, Scott LR, Munger TM. Ventricular tachycardia in the absence of structural heart disease. Indian Pacing Electrophysiol J. 2005;5(2):106-121.

18. Stenson PD, Ball EV, Mort M, Phillips AD, Shiel JA, Thomas NS, Abeysinghe S, Krawczak M, Cooper DN. Human Gene Mutation Database (HGMD): 2003 update. Hum Mutat 2003 Jun;21(6):577-581.

19. Wang K, Li M, Hakonarson H. ANNOVAR: functional annotation of genetic variants from high-throughput sequencing data. Nucleic Acids Res 2010;38(16):e164.

20. Liu X, Jian X, Boerwinkle E. dbNSFP v2.0: a database of human non-synonymous SNVs and their functional predictions and annotation. Hum Mutat 2013;34(9):E2393-2402.

21. Richards S, Aziz N, Bale S, Bick D, Das S., Gastier-Foster J, Grody W, Hegde M, Lyon E, Spector E, Voelkerding K, Rehm H. L. (2015). Standards and guidelines for the interpretation of sequence variants: a joint consensus recommendation of the American College of Medical Genetics and Genomics and the Association for Molecular Pathology. Genetics in medicine, 17(5), 405-423.

22. ClinVar, http://www.ncbi.nlm.nih.gov/clinvar

23. Kopanos, C., Tsiolkas, V., Kouris, A., Chapple, C. E., Aguilera, M. A., Meyer, R., \& Massouras, A. VarSome: the human genomic variant search engine. Bioinformatics 2019; 35(11), 1978.

24. Bekbossynova M, Akilzhanova A, Guelly C, Abilova Z, Akhmetova A, Kairov U et al. Genetic Epidemiologyof Ventricular Tachycardia in Patients with Cardiomyopathy in Kazakhstan: A Targeted Sequencing Study. Journal of Heart and Lung Transplantation. 2018 Apr 1;37(4):S322. https://doi.org/10.1016/j.healun.2018.01.820

25. Akilzhanova A. R., Guelly C., Abilova Z., Rakhimova S., Akhmetova A., Kairov U., Nuralinov O., Rashbayeva G., Trajanoski S., Zhumadilov Z., Bekbossynova M. P05.72D Mutation spectrum in a Kazakhstani cohort with ventricular tachycardia: targeted sequencing study Abstracts from the $51^{\text {st }}$ European Society of Human Genetics Conference: Posters https://www.nature.com/articles/s41431-019-0404-7

Peer) reviewing PDF | (2020:03:47033:2:0:NEW 26 Nov 2020) 
632

633

634

635

636

637

638

639

640

641

642

643

644

645

646

647

648

649

650

651

652

653

654

655

656

657

658

659

660

661

662

663

664

665

666

667

668

669

670

26. Frese KS, Katus HA, Meder B. Next-generation sequencing: from understanding biology to personalized medicine. Biology (Basel) 2013;2:378-398.

27. Roberts R, Marian AJ, Dandona S, Stewart AF. Genomics in cardiovascular disease. J Am Coll Cardiol 2013;61(20):2029-2037.

28. Schrijver I, Aziz N, Farkas DH, Furtado M, Gonzalez AF, Greiner TC, Grody WW, Hambuch T, Kalman L, Kant JA, Klein RD, Leonard DG, Lubin IM, Mao R, Nagan N, Pratt VM, Sobel ME, Voelkerding KV, Gibson JS. Opportunities and challenges associated with clinical diagnostic genome sequencing: a report of the Association for Molecular Pathology. J Mol Diagn. 2012 Nov;14(6):525-40.

29. Qian L, Bodmer R. Probing the polygenic basis of cardiomyopathies in drosophila. J Cell Mol Med 2012;16(5):972-977.

30. Lopes LR, Zekavati A, Syrris P, Hubank M, Giambartolomei C, Dalageorgou C, Jenkins S, McKenna W; Uk10k Consortium, Plagnol V, Elliott PM. Genetic complexity in hypertrophic cardiomyopathy revealed by high-throughput sequencing. J Med Genet 2013;50(4):228-239.

31. Kapa S, Tester DJ, Salisbury BA, Harris-Kerr C, Pungliya MS, Alders M, Wilde AA, Ackerman MJ. Genetic testing for long-QT syndrome: Distinguishing pathogenic mutations from benign variants. Circulation 2009;120(18):1752-1760.

32. Stattin EL, Boström IM, Winbo A, Cederquist K, Jonasson J, Jonsson BA, Diamant UB, Jensen SM, Rydberg A, Norberg A. Founder mutations characterise the mutation panorama in 200 swedish index cases referred for long qt syndrome genetic testing. BMC Cardiovasc Disord 2012;12:95.

33. Allegue C, Coll M, Mates J, Campuzano O, Iglesias A, Sobrino B, Brion M, Amigo J, Carracedo A, Brugada P, Brugada J, Brugada R. Genetic analysis of arrhythmogenic diseases in the era of NGS: The complexity of clinical decision-making in Brugada syndrome. PLoS One 2015;10(7):e0133037.

34. Herman DS, Lam L, Taylor MR, Wang L, Teekakirikul P, Christodoulou D, Conner L, DePalma SR, McDonough B, Sparks E, Teodorescu DL, Cirino AL, Banner NR, Pennell DJ, Graw S, Merlo M, Di Lenarda A, Sinagra G, Bos JM, Ackerman MJ, Mitchell RN, Murry CE, Lakdawala NK, Ho CY, Barton PJ, Cook SA, Mestroni L, Seidman JG, Seidman CE. Truncations of titin causing dilated cardiomyopathy. N Engl J Med. 2012;366(7):619-28. doi: 10.1056/NEJMoa1110186.

35. Golbus JR, Puckelwartz MJ, Fahrenbach JP, Dellefave-Castillo LM, Wolfgeher $\mathrm{D}, \mathrm{McNally}$ EM. Population-based variation in cardiomyopathy genes. Circ Cardiovasc Genet. 2012;5(4):391-9. doi: 10.1161/CIRCGENETICS.112.962928.

36. Bit-Avragim N, Perrot A, Schöls L, Hardt C, Kreuz FR, Zühlke C, Bubel S, Laccone F, Vogel HP, Dietz R, Osterziel KJ. The GAA repeat expansion in intron 1 of the frataxin gene is related to the severity of cardiac manifestation in patients with Friedreich's ataxia. J Mol Med (Berl). 2001;78(11):626-32. 
671 37. Carboni N, Marrosu G, Porcu M, Mateddu A, Solla E, Cocco E, Maioli MA, Oppo V, Piras

672

673

674

675

676

677

678

679

680

681

682

683

684

685

686

687

688

689 R, Marrosu MG. Dilated cardiomyopathy with conduction defects in a patient with partial merosin deficiency due to mutations in the laminin- $\alpha 2$-chain gene: a chance association or a novel phenotype? Muscle Nerve. 2011;44(5):826-8. doi: 10.1002/mus.22228.

38. Gollob MH, Green MS, Tang AS, Gollob T, Karibe A, Ali Hassan AS, Ahmad F, Lozado R, Shah G, Fananapazir L, Bachinski LL, Roberts R. Identification of a gene responsible for familial Wolff-Parkinson-white syndrome. N Engl J Med 2001;344(24):1823-1831.

39. Gollob MH, Green MS, Tang AS, Roberts R. PRKAG2 cardiac syndrome: Familial ventricular preexcitation, conduction system disease, and cardiac hypertrophy. Curr Opin Cardiol. 2002;17(3):229-234.

40. Aggarwal V, Dobrolet N, Fishberger S, Zablah J, Jayakar P, Ammous Z. PRKAG2 mutation: An easily missed cardiac specific non-lysosomal glycogenosis. Ann Pediatr Cardiol 2015;8(2):153-156.

41. Arad M, Benson DW, Perez-Atayde AR, McKenna WJ, Sparks EA, Kanter RJ, McGarry K, Seidman JG, Seidman CE. Constitutively active AMP kinase mutations cause glycogen storage disease mimicking hypertrophic cardiomyopathy. J Clin Invest 2002;109(3):357-362.

42. Yang Y, Fan C, Deng C, Zhao L, Hu W, Di S, Ma Z, Zhang Y, Qin Z, Jin Z, Yan X, Jiang S, Sun Y, Yi W. Melatonin reverses flow shear stress-induced injury in bone marrow mesenchymal stem cells via activation of AMP-activated protein kinase signaling. J Pineal Res 2016;60(2):228-241.

Peer] reviewing PDF | (2020:03:47033:2:0:NEW 26 Nov 2020) 


\section{Table $\mathbf{1}$ (on next page)}

Patient characteristics

CHD - coronary heart disease; DCM - dilated cardiomyopathy; VT - ventricular tachycardia, iVT - idiopathic ventricular tachycardia; CM - cardiomyopathy; SCD - sudden cardiac death; LVEF - left ventricle ejection fraction; LA - Left atrial dimension; LV EDD - Left ventricular enddiastolic dimension; LV ESD - Left ventricular end-systolic dimension. 


\section{Table 1:}

\section{Patient characteristics}

\begin{tabular}{|c|c|c|c|}
\hline Characteristics & $\begin{array}{c}\text { CHD VT, } \\
n=23\end{array}$ & $\begin{array}{c}\text { DCM VT, } \\
\mathrm{n}=32\end{array}$ & $\begin{array}{l}\mathrm{iVT}, \\
\mathrm{n}=37\end{array}$ \\
\hline Age, years & $62.3 \pm 8.8$ & $43.0 \pm 13.3$ & $37.1 \pm 19.2$ \\
\hline Sex, F/M & $1 / 22$ & $11 / 21$ & $21 / 16$ \\
\hline BMI, kg/m2 & $27.9 \pm 5.5$ & $27.0 \pm 7.2$ & $24.9 \pm 5.6$ \\
\hline \multicolumn{4}{|l|}{$\begin{array}{l}\text { NYHA functional class and } \\
\text { functional parameters }\end{array}$} \\
\hline $\mathrm{I}$ & $1(4.3 \%)$ & $1(3.1 \%)$ & $25(67.6 \%)$ \\
\hline II & $6(26.1 \%)$ & $1(3.1 \%)$ & $11(29.7 \%)$ \\
\hline III & $16(69.6 \%)$ & $20(62.5 \%)$ & $1(2.7 \%)$ \\
\hline IV & $0(0 \%)$ & $10(31.2 \%)$ & $0(0 \%)$ \\
\hline LVEF, \% & $36.6 \%$ & $25.5 \%$ & $60.9 \%$ \\
\hline $\mathrm{LA}, \mathrm{mm}$ & $42.9 \pm 6.2$ & $47.3 \pm 7.8$ & $30.6 \pm 6.7$ \\
\hline LV EDD, $\mathrm{cm}$ & $6.2 \pm 1.0$ & $6.9 \pm 0.8$ & $4.6 \pm 0.8$ \\
\hline LV ESD, cm & $5.1 \pm 1.4$ & $6,0 \pm 0,9$ & $3.1 \pm 0.9$ \\
\hline QRS Interval, ms & $112.4 \pm 29.7$ & $117.4 \pm 27.3$ & $89.9 \pm 15.4$ \\
\hline $\begin{array}{l}\text { QT Interval, ms } \\
\end{array}$ & $401.5 \pm 72.0$ & $389.0 \pm 38.0$ & $400.5 \pm 44.8$ \\
\hline \multicolumn{4}{|l|}{ Family history of CM or SCD } \\
\hline $\begin{array}{l}\text { familial } \\
\end{array}$ & $6(26.1 \%)$ & $8(25 \%)$ & $11(29.7 \%)$ \\
\hline sporadic & $17(73.9 \%)$ & $24(75 \%)$ & $24(64.9 \%)$ \\
\hline unknown & 0 & 0 & $2(5.4 \%)$ \\
\hline \multicolumn{4}{|c|}{$\begin{array}{l}\text { CHD - coronary heart disease; DCM - dilated cardiomyopathy; VT - ventricular tachycardia, } \\
\text { iVT - idiopathic ventricular tachycardia; CM - cardiomyopathy; SCD - sudden cardiac death; } \\
\text { LVEF - left ventricle ejection fraction; LA - Left atrial dimension; LV EDD - Left ventricular } \\
\text { end-diastolic dimension; LV ESD - Left ventricular end-systolic dimension. }\end{array}$} \\
\hline
\end{tabular}

3 


\section{Table 2 (on next page)}

Statistical testing (Kruskal-Wallis Test) of clinical parameters

CHD - coronary heart disease; DCM - dilated cardiomyopathy; VT - ventricular tachycardia, iVT - idiopathic ventricular tachycardia; CM - cardiomyopathy; SCD - sudden cardiac death; LVEF - left ventricle ejection fraction; LA - Left atrial dimension; LV EDD - Left ventricular enddiastolic dimension; LV ESD - Left ventricular end-systolic dimension. 
1 Table 2:

2 Statistical testing (Kruskal Wallis Test) of clinical parameters

\begin{tabular}{|c|c|c|c|c|c|c|c|c|}
\hline & Groups & $\mathrm{N}$ & Median & $\begin{array}{c}25- \\
\text { Percentile }\end{array}$ & $\begin{array}{c}\text { 75- } \\
\text { Percentile }\end{array}$ & $\begin{array}{l}\mathrm{p} \text {-value } \\
\text { overall } \\
\text { test }\end{array}$ & $\begin{array}{c}\text { Pairwise } \\
\text { comparison }\end{array}$ & $\begin{array}{l}\mathrm{p}_{\text {adj- }} \\
\text { value }\end{array}$ \\
\hline \multirow{3}{*}{$\begin{array}{l}\text { LVEF, } \\
\%\end{array}$} & CHD VT & 23 & 35.00 & 26.13 & 45.00 & \multirow{3}{*}{0.000} & $\begin{array}{l}\text { DCM VT - CHD } \\
\text { VT }\end{array}$ & 0.067 \\
\hline & DCM VT & 32 & 24.50 & 18.25 & 30.00 & & DCM VT - iVT & 0.000 \\
\hline & iVT & 37 & 61.29 & 57.13 & 67.48 & & CHD VT - iVT & 0.000 \\
\hline \multirow{3}{*}{$\begin{array}{l}\mathrm{LA}, \\
\mathrm{mm}\end{array}$} & CHD VT & 23 & 44.00 & 37.00 & 46.00 & \multirow{3}{*}{0.000} & $\begin{array}{l}\text { DCM VT - CHD } \\
\text { VT }\end{array}$ & 0.537 \\
\hline & DCM VT & 32 & 45.50 & 42.00 & 49.75 & & DCM VT - iVT & 0.000 \\
\hline & iVT & 37 & 31.00 & 26.60 & 35.00 & & CHD VT - iVT & 0.000 \\
\hline \multirow{3}{*}{$\begin{array}{l}\mathrm{LV} \\
\mathrm{EDD} \\
\mathrm{cm}\end{array}$} & CHD VT & 23 & 6.10 & 5.70 & 6.60 & \multirow{3}{*}{0.000} & $\begin{array}{l}\text { DCM VT - CHD } \\
\text { VT }\end{array}$ & 0.153 \\
\hline & DCM VT & 32 & 6.87 & 6.33 & 7.45 & & DCM VT - iVT & 0.000 \\
\hline & iVT & 37 & 4.70 & 4.22 & 4.97 & & CHD VT - iVT & 0.000 \\
\hline \multirow{3}{*}{$\begin{array}{c}\mathrm{LV} \\
\mathrm{ESD}, \\
\mathrm{cm}\end{array}$} & CHD VT & 23 & 4.80 & 4.00 & 5.90 & \multirow{3}{*}{0.000} & $\begin{array}{l}\text { DCM VT - CHD } \\
\text { VT }\end{array}$ & 0.099 \\
\hline & DCM VT & 32 & 5.92 & 5.63 & 6.50 & & DCM VT - iVT & 0.000 \\
\hline & iVT & 37 & 3.20 & 2.63 & 3.50 & & CHD VT - iVT & 0.000 \\
\hline \multirow{3}{*}{$\begin{array}{c}\text { QRS } \\
\text { Interval } \\
, \mathrm{ms}\end{array}$} & CHD VT & 23 & 104.00 & 98.00 & 122.00 & \multirow{3}{*}{0.000} & $\begin{array}{l}\text { DCM VT - CHD } \\
\text { VT }\end{array}$ & 1.000 \\
\hline & DCM VT & 32 & 113.00 & 98.50 & 122.00 & & DCM VT - iVT & 0.000 \\
\hline & iVT & 37 & 86.00 & 80.00 & 97.00 & & CHD VT - iVT & 0.001 \\
\hline \multirow{3}{*}{$\begin{array}{c}\mathrm{QT} \\
\text { Interval } \\
, \mathrm{ms}\end{array}$} & CHD VT & 23 & 400.00 & 374.00 & 450.00 & \multirow{3}{*}{0.203} & & \\
\hline & DCM VT & 32 & 394.00 & 363.00 & 403.50 & & & \\
\hline & iVT & 37 & 400.00 & 380.00 & 427.00 & & & \\
\hline \multicolumn{9}{|c|}{$\begin{array}{l}\text { CHD - coronary heart disease; DCM - dilated cardiomyopathy; VT - ventricular tachycardia, iVT } \\
\text { - idiopathic ventricular tachycardia; CM - cardiomyopathy; SCD - sudden cardiac death; LVEF - } \\
\text { left ventricle ejection fraction; LA - Left atrial dimension; LV EDD - Left ventricular end- } \\
\text { diastolic dimension; LV ESD - Left ventricular end-systolic dimension. }\end{array}$} \\
\hline
\end{tabular}




\section{Table 3 (on next page)}

Frequency of patients positive for pathogenetic variants in the clinical subgroups

*Variants per positive patient was calculated by dividing the (cumulating) number of variants by the number of positive patients. Class I (highest pathogenic potential) variants were predicted disease causing by at least 7 of the tools, class II (intermediate pathogenic potential) variants were predicted disease causing by 4-6 of the tools, class III (low pathogenic potential) were predicted disease causing by 1-3 prediction tools, and class IV (benign) was predicted disease causing by none of the tools (0). 
1 Table 3:

2 Frequency of patients positive for pathogenetic variants in the clinical subgroups

\begin{tabular}{|l|c|r|r|r|r|}
\hline & $\begin{array}{l}\text { patients } \\
\text { carrying } \geq 1 \\
\text { class I variant }\end{array}$ & \% positive & $\begin{array}{l}\text { cumulative } \\
\text { number of } \\
\text { variants }\end{array}$ & $\begin{array}{l}\text { \% of all } \\
\text { variants }\end{array}$ & $\begin{array}{l}\text { variants per } \\
\text { positive } \\
\text { patient* }\end{array}$ \\
\hline CHD VT $(n=23)$ & 10 & $43.5 \%$ & 13 & $11.8 \%$ & 1.3 \\
\hline DCM VT $(n=32)$ & 10 & $31.3 \%$ & 13 & $8.2 \%$ & 1.3 \\
\hline iVT $(n=37)$ & 20 & $54.1 \%$ & 26 & $13.9 \%$ & 1.3 \\
\hline & $\begin{array}{c}\text { 1 class I/II } \\
\text { variant }\end{array}$ & & & & \\
\hline CHD VT $(n=23)$ & 16 & $69.6 \%$ & 31 & $28.2 \%$ & 1.94 \\
\hline DCM VT $(n=32)$ & 24 & $75.0 \%$ & 49 & $30.8 \%$ & 2.04 \\
\hline iVT $(n=37)$ & 30 & $81.1 \%$ & 70 & $37.4 \%$ & 2.33 \\
\hline & 1 class & & & & \\
\hline CHD VT $(n=23)$ & 22 & $95.7 \%$ & 89 & $80.9 \%$ & 4.05 \\
\hline DCM VT $(n=32)$ & 31 & $96.9 \%$ & 131 & $82.4 \%$ & 4.23 \\
\hline iVT $(n=37)$ & $97.3 \%$ & 160 & $85.6 \%$ & 4.44 \\
\hline
\end{tabular}

*Variants per positive patient was calculated by dividing the (cumulating) number of variants by the number of positive patients.

Class I (highest pathogenic potential) variants were predicted disease causing by at least 7 of the tools, class II (intermediate pathogenic potential) variants were predicted disease causing by 4-6 of the tools, class III (low pathogenic potential) were predicted disease causing by 1-3 prediction tools, and class IV (benign) was predicted disease causing by none of the tools $(0)$. 


\section{Table 4 (on next page)}

Distribution of class I-IV variants according to their molecular function/association

Class I (highest pathogenic potential) variants were predicted disease causing by at least 7 of the tools, class II (intermediate pathogenic potential) variants were predicted disease causing by 4-6 of the tools, class III (low pathogenic potential) were predicted disease causing by 1-3 prediction tools, and class IV (benign) was predicted disease causing by none of the tools (0). 
1 Table 4:

2 Distribution of class I-IV variants according to their molecular function/association

3

\begin{tabular}{|l|c|c|c|c|}
\cline { 2 - 5 } \multicolumn{1}{c|}{} & Class I & Class II & Class III & Class IV \\
\hline Cell membrane & $0(0 \%)$ & $7(41.2 \%)$ & $8(47.1 \%)$ & $2(11.8 \%)$ \\
\hline Cytoskeleton & $5(9.8 \%)$ & $12(23.5 \%)$ & $22(43.1 \%)$ & $12(23.5 \%)$ \\
\hline Sarcomere & $14(11.7 \%)$ & $34(18.3 \%)$ & $62(51.7 \%)$ & $10(8.3 \%)$ \\
\hline Metabolism & $4(23.5 \%)$ & $2(11.8 \%)$ & $10(58.8 \%)$ & $1(5.9 \%)$ \\
\hline Intercalated disc & $6(20.0 \%)$ & $5(16.7 \%)$ & $15(50.0 \%)$ & $4(13.3 \%)$ \\
\hline Ion flux & $12(30.8 \%)$ & $7(17.9 \%)$ & $18(46.2 \%)$ & $2(5.1 \%)$ \\
\hline Nucleus & $7(21.2 \%)$ & $3(9.1 \%)$ & $21(63.6 \%)$ & $2(6.1 \%)$ \\
\hline
\end{tabular}

Class I (highest pathogenic potential) variants were predicted disease causing by at least 7 of the tools, class II (intermediate pathogenic potential) variants were predicted disease causing by 4-6 of the tools, class III (low pathogenic potential) were predicted disease causing by 1-3 prediction tools, and class IV (benign) was predicted disease causing by none of the tools (0). 
Figure 1

Distribution of HGMD listed variants and rare variants with respect to their pathogenic potential

Mean average variant frequency in public databases (ESP6500, 1000G2012apr_all, and EXAC_ALL) is 0.000279 for class I variants (high pathogenic potential), 0.00267 for class II (intermediate pathogenic potential), 0.00372 for class III (low pathogenic potential), and 0.0192 for class IV (benign) variants

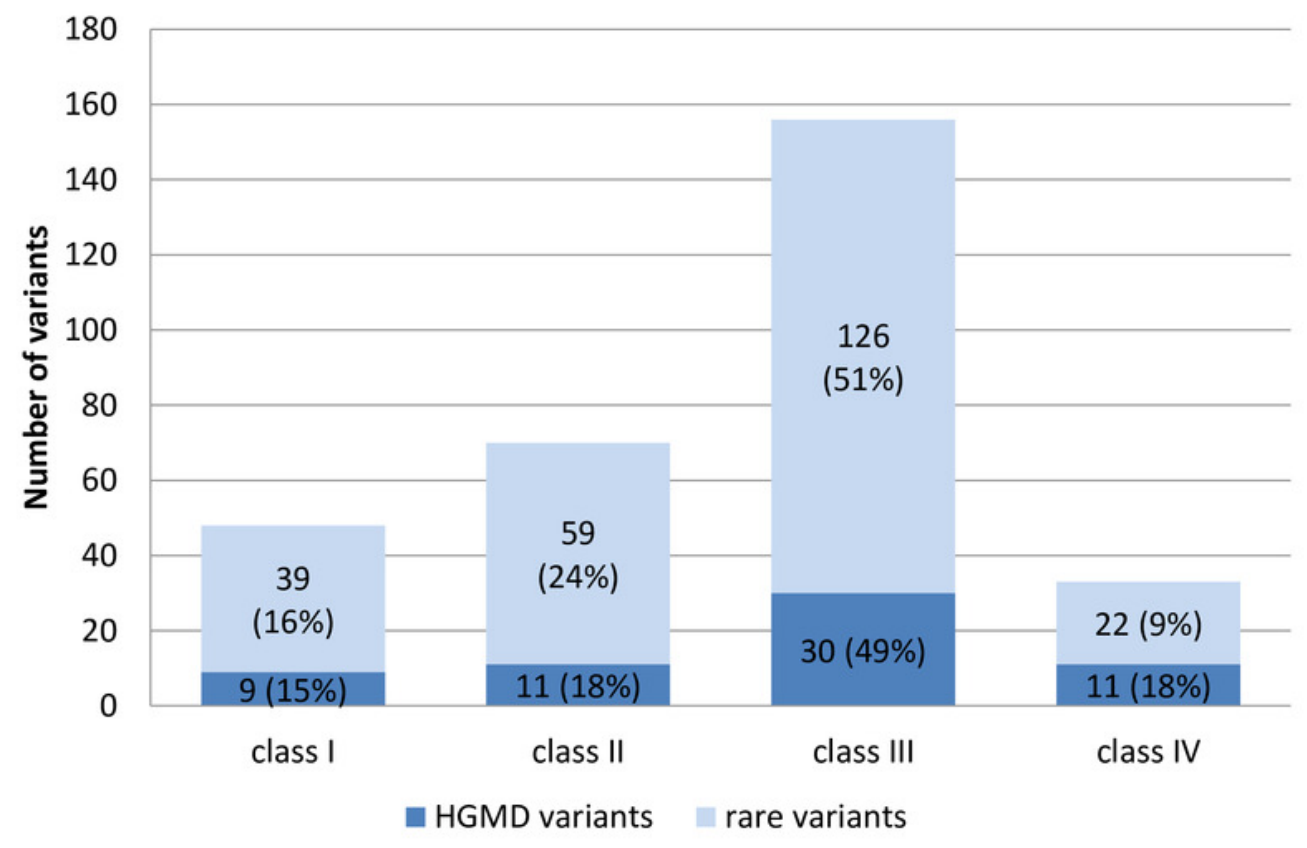


Figure 2

Frequency of HGMD mutations per clinical subgroup

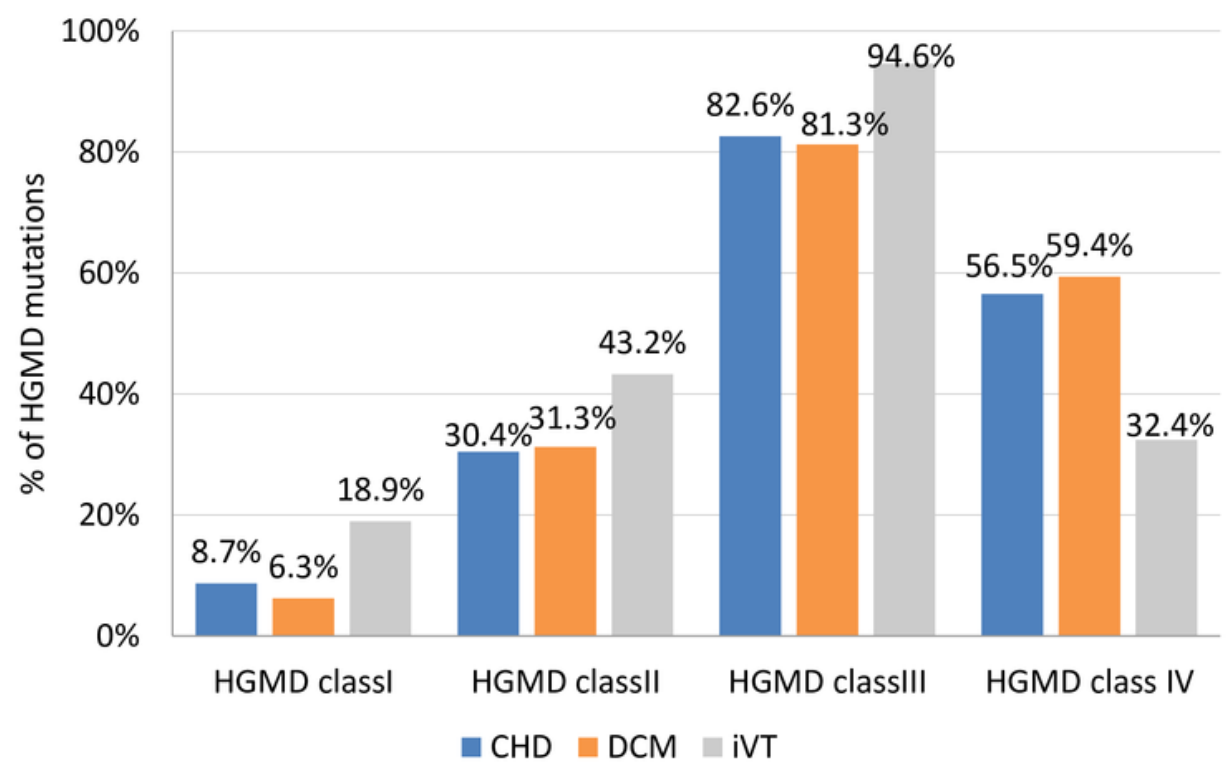


Figure 3

Distribution of variants according to ACMG guidelines among all classes of 307 genetic variants

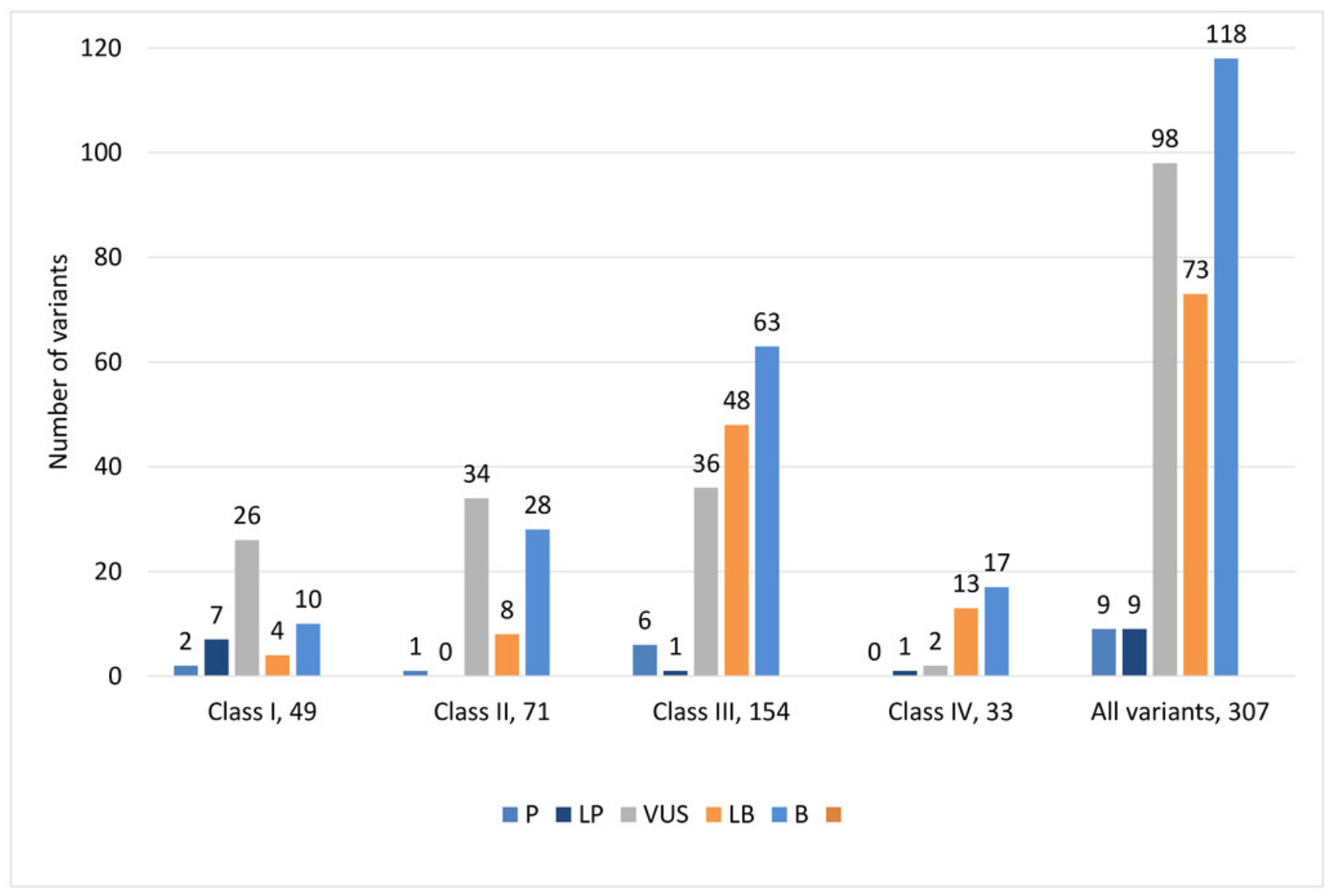


Figure 4

Distribution of variants (class I+class II) according to their molecular function/association

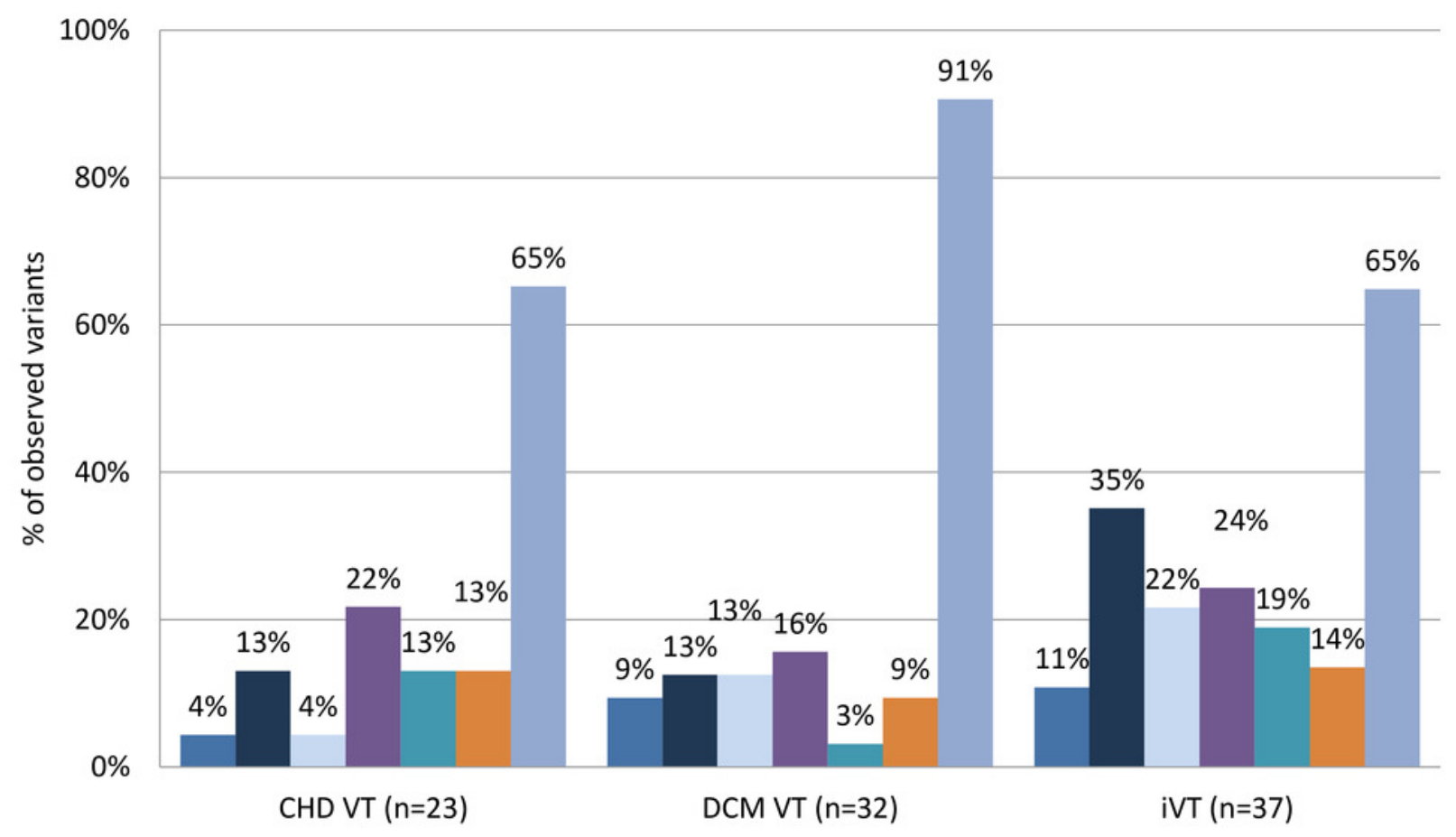

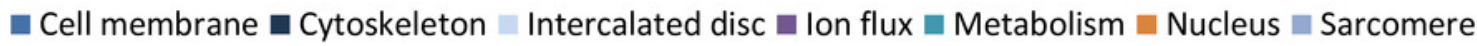


Figure 5

Frequency of HGMD plus variants within each clinical subgroup

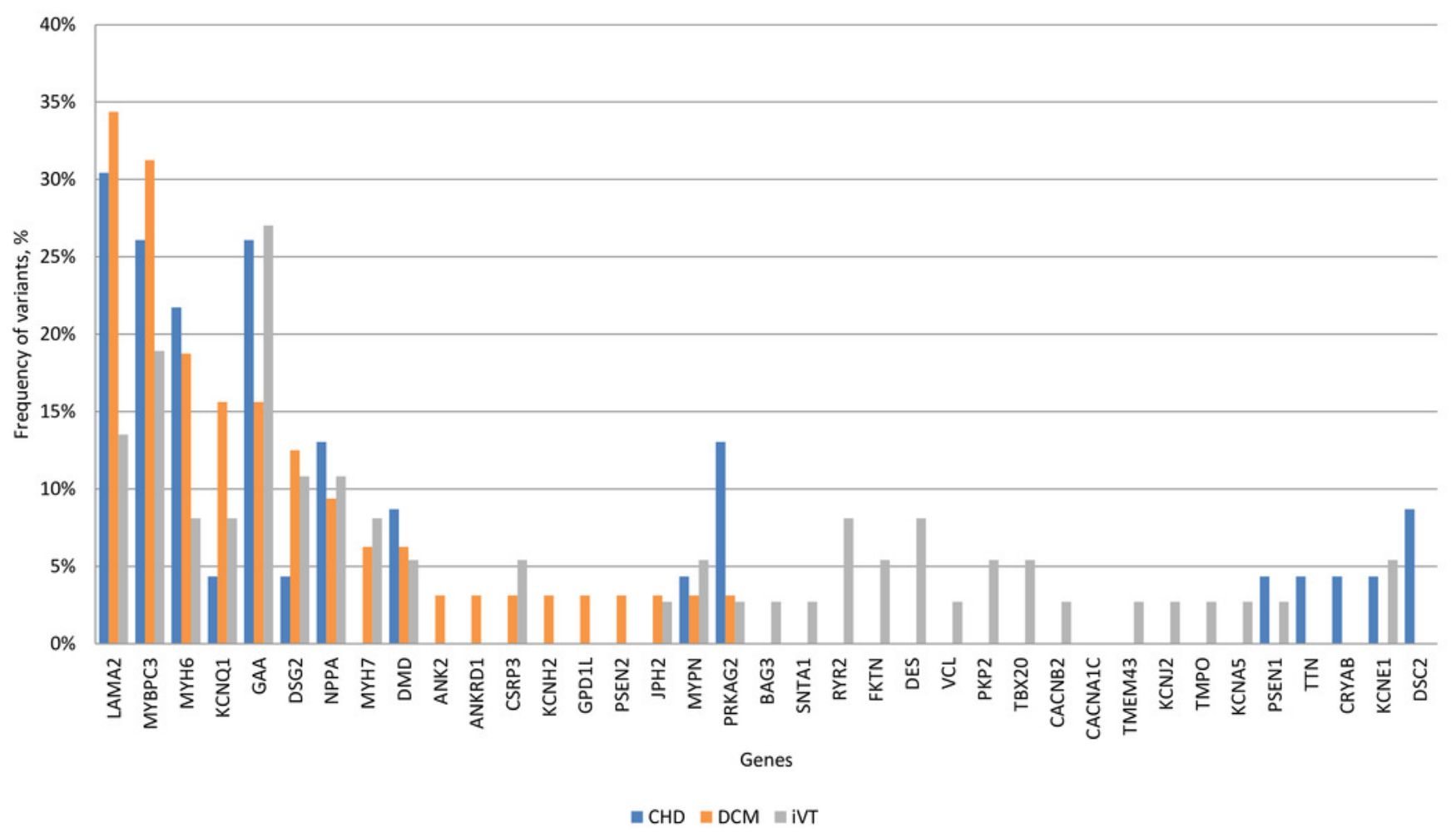

